\title{
Review suggests high survival rates for veneers at five and ten years
}

\author{
Abstracted from \\ Layton DM, Clarke M, Walton TR. \\ A systematic review and meta-analysis of the survival of feldspathic \\ porcelain veneers over 5 and 10 years. Int J Prosthodont 2012; 25: 590-603. \\ Address for correspondence: Dr Danielle M. Layton, 217 Wickham Terrace, \\ Brisbane, Queensland, Australia, 4000. E-mail: laytonpros@dlpros.com.au
}

\section{Question: How long do porcelain veneers last?}

Data sources The Cochrane Library, Medline, Embase, and Web of Knowledge databases were searched and the Evidence-Based Dentistry, International Journal of Prosthodontics, Journal of Prosthetic Dentistry, Journal of Adhesive Dentistry, Journal of Esthetic and Restorative Dentistry, and Journal of Oral Rehabilitation were hand searched. Clinical trials registers, conference proceedings and academic colleagues were contacted to identify unpublished data, abstracts and other gray literature. There were no language limits.

Study selection Article identification, screening, and eligibility and inclusion assessments were completed independently by two reviewers. Prospective and retrospective cohort and controlled trials assessing the outcome of feldspathic porcelain veneers in more than 15 patients were included. Some veneers in each study had to be in situ for five years. Veneers placed using unusual techniques, such as with no enamel preparation or excessive incisal edge lengthening, were excluded. Data extraction and synthesis Estimated cumulative survival and standard error for each study were assessed and used for meta-, sensitivity and post hoc analyses. The $\mathrm{I}^{2}$ statistic and the Cochran $Q$ test and its associated $P$ value were used to evaluate statistical heterogeneity, with a random-effects meta-analysis used when the $P$ value for heterogeneity was less than 0.1 . Heterogeneity, publication patterns and small study biases were explored.

Results Eleven studies were included with six (four prospective and two retrospective cohorts) contributing to the meta-analysis. The estimated cumulative survival for feldspathic porcelain veneers was 95.7\% (95\% confidence interval [Cl]: $92.9 \%$ to $98.4 \%$ ) at five years and ranged from $64 \%$ to $95 \%$ at ten years across three studies. A post hoc meta-analysis indicated that the 10-year best estimate may approach $95.6 \%$ (95\% Cl: $93.8 \%$ to $97.5 \%)$. High levels of statistical heterogeneity were found.

Conclusions When bonded to enamel substrate, feldspathic porcelain veneers have a very high 10-year survival rate that may approach $95 \%$. Clinical heterogeneity is associated with differences in reported survival rates. Use of clinically relevant survival definitions and careful reporting of tooth characteristics, censorship, clustering, and precise results in future research would improve meta-analytic estimates and aid treatment decisions.

\section{Commentary}

Porcelain veneers have been increasingly used to restore teeth since the 1980s when enamel etching and porcelain surface treatments improved the predictability of bonding. Patients are becoming more aware of these as both restorative and aesthetic treatment options and so are being more widely used than ever. ${ }^{1}$ In 2011/12, 14,249 veneers were placed in primary care NHS dental practices in Scotland (with an item of service value of $£ 1,537,695) .^{2}$ There is limited evidence about the long-term success of these restorations in the NHS in Scotland (or indeed elsewhere), although the proposed re-introduction of tooth specific information is likely to provide data for future studies. ${ }^{3}$

The aim of this paper was to quantify the long-term survival of feldspathic porcelain veneers from a systematic review and metaanalysis of the international literature. Clear aims were set out and a focused search included: feldspathic porcelain veneers in studies with greater than fifteen patients, and at least some of the veneers had to be present for five years. The search for studies was thorough as they searched for unpublished as well as published studies and included non-English studies in their search. However, they could perhaps have included other databases such as PubMed, Web of Science and Google Scholar and formally included citation searching. Six studies were eventually included in the meta-analysis: two were retrospective cohorts and four were prospective cohorts. No randomised controlled trials were available which would have provided more robust data. The quality appraisal of studies was also not as comprehensive as it could have been. Specific relevant study quality factors were not assessed such as sample size, power and study inclusion criteria. The study characteristics were presented in a clear table, however the studies were variable in their design and had defined survival differently - resulting in significant heterogeneity. The authors addressed this variability to improve consistency of outcomes by standardising outcome criteria across studies.

The results provide favourable survival rates for feldspathic porcelain veneers; estimated cumulative survival was 95.7\% (95\% CI: 92.9 to 98.4$)$ at five years and a post hoc analysis indicated that the 10-year results may approach 95.6\% (95\% CI: 93.8 to 97.5 ). The results appear to be quite precise with tight confidence intervals. However the paper does not look into other factors which will effect the success of veneers for example: age of patient, experience of practitioner, type of treatment payment, carious versus no carious cavities, extent into enamel/dentine. Therefore caution should be used on applying the results and considering its outcomes. The authors acknowledge that more research is required with 'careful 


\section{RESTORATIVE DENTISTRY}

reporting of tooth characteristics, censorship, clustering and precise results to improve the meta-analysis and aid treatment decisions'.

It is mindful to be aware of these results when discussing treatment options with patients. Informed consent should give them an idea of risks, benefits, costing and longevity of restorations. These results can give an indication of longevity of feldspathic veneers. Care must also be taken as different types of veneers are also becoming more widely available eg pressed laminate, milled zirconia - but similar long-term comparative data are more limited, which would help provide information on best practice.
Eileen Watt ${ }^{1}$ and David I Conway ${ }^{2}$

${ }^{1}$, Glasgow Dental Hospital and School; ${ }^{2}$, University of Glasgow

Dental School, Glasgow, UK

1. McLaren EA, LeSage B. Feldspathic veneers: what are their indications? Compend Contin Educ Dent 2011; 32: 44-49.

2. Information Services Division. Dental Care Information. NHS National Services Scotland, Edinburgh, 2012. http://www.isdscotland.org/Health-Topics/Dental-Care/ Accessed March 2013.

3. Chief Dental Officer and Dentistry Division. General Dental Services - Introduction of tooth specific information. NHS PCA(D)(2012)6 http://www.sehd.scot.nhs.uk/pca/ PCA2012\%28D\%2906.pdf Accessed March 2013.

Evidence-Based Dentistry (2013) 14, 15-16. doi:10.1038/sj.ebd.6400914 